\title{
The Best Choice to Achieve Zero Complications after Pancreatoduodenectomy
}

\author{
Shinji Osada, Hisashi Imai, Yoshiyuki Sasaki, Itaru Yasufuku, Ryuichi Asai, \\ Yoshihisa Tokumaru, Takuji Sakuratani, Kazuhiro Yoshida \\ Surgical Oncology, Gifu University School of Medicine, Gifu, Japan \\ E-mail: sting@gifu-u.ac.jp \\ Received September 30, 2010; revised January 21, 2011; accepted January 26, 2011
}

\begin{abstract}
Pancreatoduodenectomy (PD) has been performed commonly, but the occurrence of pancreatic fistula (PF) is a critical trigger of complications, which are potentially life threatening, and is also associated with markedly prolonged hospitalization. Many techniques have been proposed for connecting the pancreatic stump with the gastrointestinal tract, stomach vs. jejunum, etc. Among the risk factors for PF, such as general patient factors or disease-related factors, the most important is the texture of the remnant pancreas. Surgical technique might be one improvable aspect that can reduce the pancreatic leakage rate, therefore; various methods of managing the pancreatic remnant have been studied. Methods of reconstruction between the remnant pancreas and the intestine include end-to-side with/without duct-to-mucosa anastomosis or end-to-end invagination styles, has been argued. Here, we review several trials for safety and methods of treating the pancreatic stump after PD, and demonstrate our experiences.
\end{abstract}

Keywords: Pancreatoduodenectomy, Reconstruction, Pancreatojejunostomy, Pancreatic Fistula, Pancreatoenteric Anastomosis

\section{Introduction}

The indications for pancreatoduodenectomy (PD) have expanded to encompass a broad spectrum of periampullary tumors including both malignant and benign lesions, chronic pancreatitis, and, occasionally, trauma. The occurrence of pancreatic fistula (PF) is a critical trigger of complications such as intra-abdominal abscess and hemorrhage, which are potentially life threatening, and is also associated with markedly prolonged hospitalization. Many techniques have been proposed for connecting the pancreatic stump with the gastrointestinal tract but despite some success, problems remain, especially with a soft pancreas gland with a small duct [1]. Most of the large PD series have reported rates of PF of over $10 \%$ [1-4]. Risk factors for PF depend upon 1) general patient factors, including age, sex, jaundice and nutrition; 2) disease-related factors, including pancreatic duct size, pancreatic texture, and pathology; and 3) procedure-related factors, including blood loss, operative time, and anastomotic method [5]. Among these risk factors, the most important might be the texture of the remnant pancreas. Indeed, the occurrence of PF rises to nearly $20 \%$ in cases of soft pancreatic texture, despite an occurrence rate of $5 \%$ in cases of hard pancreatic tissue [2-4]. Because surgical technique might be one improvable aspect that can reduce the pancreatic leakage rate, various methods of managing the pancreatic remnant have been studied. Methods of reconstruction between the remnant pancreas and the intestine include end-to-side with/ without duct-to-mucosa anastomosis or end-to-end invagination styles, and arguably, anastomosis of the remnant pancreas with the stomach is also another method. Here, we review several trials for safety and certain methods of treating the pancreatic stump after PD.

\section{Anastomosis Methods with Pancreas}

\subsection{Pancreatojejunostomy}

PD consists of mainly two types of reconstruction procedures for the remnant alimentary tract: Billroth I (Imanaga method) with gastrojejunostomy, pancreatojejunostomy, and choledochojejunostomy; and Billroth II (Whipple and/or Child method) with pancreatojejunostomy or choledochojejunostomy and gastrojeju- 
nostomy. Billroth I reconstruction has been most commonly performed because it is conceivable that the passage of food through the entire remnant upper small intestine is more physiologically normal, and the mixture of food with bile is similar to that in normal subjects. In fact, hepatobiliary and gastrointestinal dual scintigraphy has demonstrated satisfactory mixing of bile and food [6]. Billroth I reconstruction also enables endoscopic study of the patency of the pancreatic and bile ducts in conjunction with evaluation of exocrine function [7]. However, another study demonstrated no benefit to nutritional status and quality of life after Billroth I [8]. Furthermore, in consideration of early postoperative complications, more frequent anastomotic failure of the pancreatojejunostomy using the Billroth I method has been reported than with other procedures [9]. Although the cause of this failure remains unclear, the angularity of the jejunal loop might be related to these problems [10] and, as described previously, we suspect the mixture of bile and pancreatic juice-induced enzyme activation to be associated with damage to the tissue [11]. Delayed gastric emptying (DGE), which is critical for the determination of nutritional status, has also been a concern following Billroth I reconstruction. The cause of DGE has been indicated to relate not only to anastomotic leakage [12] but also to disruption of the gastroduodenal neural connection by PD or to residual pancreatic fibrosis [13]. However, a disadvantage of Billroth II reconstruction is that bile leakage tends to occur more frequently [14]. In fact, biliogastric reflux after the type II operation is problematic in the late postoperative period [15]. As a consequence, abnormal motility of the afferent jejunum due to dietary moderation induces bile status, resulting in a high prevalence of bile leakage.

Continuous duct-to-mucosa anastomosis was described as being safer and as having a significantly lower leakage rate $[16,17]$. However, a prospective randomized clinical trial found it to be favored in low-risk patients with a dilated pancreas duct or firm fibrotic pancreas, whereas the invagination technique was better for highrisk patients with small ducts or a soft friable pancreas [18]. In addition, in an analysis of the occurrence of PF in pancreatojejunostomy, $40 \%$ originated from the parenchyma or a small side branch duct and appeared to be as common as duct-to-mucosa anastomosis [19]. In particular, for a soft pancreas, no pancreatic duct dilatation is usually detected; thus, duct-to-mucosa anastomosis might be difficult.

\subsection{Pancreatogastrostomy}

Since several retrospective studies reported that pancreatogastrostomy (PG) reduces the occurrence of PF after PD [20,21], there has been trend toward increasing use of this type of anastomosis. However, the results of a prospective randomized trial comparing PG with pancreatojejunostomy showed that the overall incidence of PF was $11.7 \%$, and the condition occurred with similar frequency after pancreatojejunostomy (11.1\%) and after PG (12.3\%) [20,22]. Length of postoperative hospital stay also did not differ between the two procedures. Because the objective safety of PG was not supported by the data from these prospective studies and meta-analysis [23], the best method for dealing with the pancreatic stump after PD remains in question. In addition, disadvantages of PG have been identified, including an increased incidence of DGE and of pancreatic duct obstruction due overgrowth by the gastric mucosa [13]. In theory, PG has several advantages over pancreatojejunostomy as the preferred method of reconstruction after PD. First, a low incidence of pancreatic leakage seems likely because the anastomosis is made with the thick and richly vascular gastric wall. Second, PG is known to suppress activation of proteolytic enzymes. Enterokinase in particular is required to convert trypsinogen to the active form, trypsin, and is present in small intestine mucosa but not in gastric mucosa. This activation also requires a neutral $\mathrm{pH}$. Therefore, even if leakage does occur, it does not lead to life-threatening complications because the pancreatic enzymes are hardly activated. Indeed, a comparative clinical study found that PG is safer than pancreatojejunostomy, particularly with regard to the incidence of PF [20,21]. However, questions remain regarding the long-term endocrine and exocrine function of the pancreas after PG. Available data on hormone levels indicate that endocrine function appears to be equal, but exocrine function appears to be worse after PG than after pancreatojejunostomy [24], resulting in severe atrophic changes in the remnant pancreas [25]. Therefore, pancreatojejunostomy may be preferable to maintain activation of the pancreatic enzymes for more physiologic digestion and absorption.

\section{Reducing Problms of Anastomosis with the Pancreas Stump}

In the past several years, increased intraoperative blood loss has been recognized as a predictive factor for PF [26, 27]. Because an adequate blood supply to the stump of the pancreas is critical to wound healing, the next step leading to a successful anastomosis [28], postoperative infusion planning must be supported. The use of somatostatin analogue has also been focused on to prevent PF [29], but its use is still not accepted as a consensus [30, 31]. The risk of developing a PF is known to be significantly associated with the final histopathological diagno- 
sis of the resected specimen, with lower risk in adenocarcinoma and higher risk in cystic neoplasms or disease originating from the bile duct $[32,33]$. This might be due to the fact that pancreatic adenocarcinoma usually occludes the main pancreatic duct, causing duct dilatation and distal inflammation. Included as a possibility is cancer occurring in chronic pancreatitis, where a fibrotic hard remnant pancreas is easily anastomosed, but a soft pancreas remains at risk of PF due to its fragility and its secretion of a high amount of pancreatic juice [18].

Many surgeons have used a stent across the pancreaticoenterostomy to prevent $\mathrm{PF}$, and a stent may be useful for diversion of pancreatic juice from the pancreatic anastomotic site, decompression of the remnant pancreas, and patency of the main pancreatic duct. Reported findings show no significant difference between internal and external stenting [34], whereas placement of drainage tube was associated with a clearly lower PF rate compared with nonstented patients [3]. Due to the concern about length of hospital stay, shorter postoperative length is not only considered a predictor of less-invasive surgical procedures but also forces evaluation of the necessity of wound treatment or external tube placement. Potential complications associated with stent removal are also argued because local peritonitis after stent removal has been reported [35]. To reduce postoperative complications, placement of a stent may be critical, and the internal type might be better than external, then internal lost tube might be best.

\section{Novel Modified Reconstruction Method}

\subsection{Background}

As suggested by the pathogenesis of the congenital choledochal cyst, reflux of pancreatic juice into the biliary tree could have an adverse effect on the bile duct wall. In particular, lysolecithin, which is converted from bile lecithin by pancreatic juice components including phospholipase A, causes severe cellular injury. Phospholipase A itself is activated by lysolecithin, and these enzymes strongly interact. In Child's type reconstruction, one of the most common reconstruction methods, the hepatojejunostomy site is several centimeters distal to the pancreatojejunostomy site. Once leakage develops at the hepatojejunostomy site, the presence of pancreatic juice will exacerbate the leakage problem. A similar problem occurs with the Whipple method, in which the hepatoand pancreato-jejunostomy anastomoses are reversed. Thus, the association of pancreatojejunostomy with life-threatening postoperative complications can be explained by the enzyme activation theory. Therefore, the safest type of anastomosis is one in which the mixture of pancreatic and biliary enzymes is contained, such as in a jejunojejunostomy. A novel modified type of reconstruction, the separated loop (SL) method, which prevents pancreatic leakage and critical secondary complications, has been well tolerated (Figure 1).

Biliary or pancreas duct drainage tubes are not necessary, and just one drainage tube is placed that is pulled out within 4 days after surgery to reduce intra-abdominal infection induced by long-term tube placement [36]. The full details were described previously [11].

\subsection{Outcome}

The SL method, as a Billroth II reconstruction, was evaluated at a single institution by comparison to PG or the Imanaga method, as Billroth I reconstructions, according to postoperative patient condition determined from blood test values and complications incurred [11, 37]. Of 107 patients undergoing PD, 31 were selected for PG, 26 for the Imanaga method, and 38 for the SL method. PG was achieved with an invagination anastomosis, which was constructed with two layers of interrupted sutures from an anterior gastrotomy and a pancreatic duct tube exiting through the stomach and abdominal wall.

There were no significant differences between PG and the Imanaga and SL methods in terms of mean total blood loss, operation time, or changes in patient body

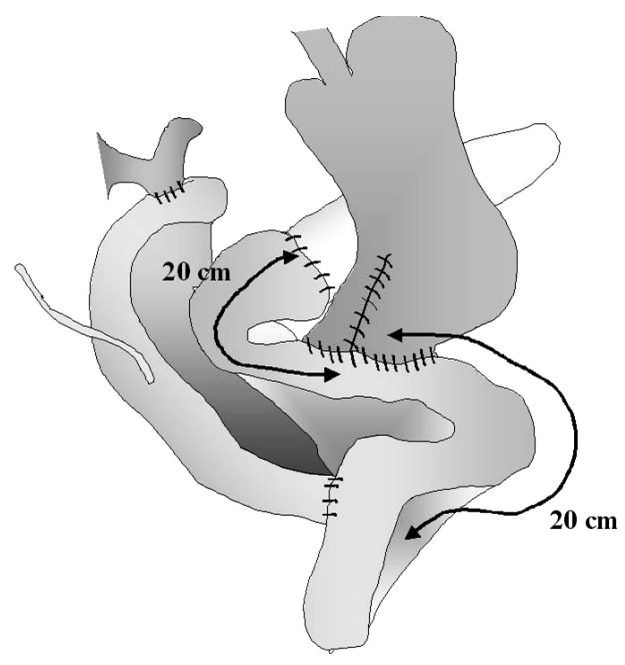

Figure 1. Schema of separated loop reconstruction method. (The jejunum is reflected upward through an incision in the transverse mesocolon, and anastomosed end-to-side with the choledochus. At $20 \mathrm{~cm}$ distal to this biliary anastomosis, the jejunum is interrupted and the end of the pancreas is inserted into the bowel by means of an invagination technique. At $20 \mathrm{~cm}$ distal to this pancreatojejunostomy, the jejunum is anastomosed to the stomach in an end-to-side fashion. Approximately $20 \mathrm{~cm}$ distal to the gastrostomy, a Y-type reconstruction of the jejunum is made with the distal end of the biliary route). 
weight. However, DGE was the most frequent cause of morbidity and was observed exclusively among patients undergoing PG (12.9\%). Of the patients undergoing the Imanaga method, $19.2 \%$ showed a high amylase level in their drainage fluid, with $3.7 \%$ mortality due to abdominal bleeding after postoperative day 52. In $6.5 \%$ and $5.2 \%$ of the patients undergoing PG or the SL method, respectively, a high amylase level was detected, but no problematic clinical events were observed. No patient required re-operation. Compared with the IM method and PG, values of postoperative blood tests were more favorable for the SL method. The postoperative condition of our patients who underwent SL reconstruction was good, suggesting that this method reduces the incidence of serious complications immediately after surgery. In the SL method, suturing of the anterior outer layer can lead to pancreas injury, especially with soft pancreas tissue; therefore, in recent cases, the anterior layer is made in single for incomplete invagination (Figure 2). Before beginning anterior layer suturing, two transpancreatic U-sutures are placed with 4-0 PDS suture. The U-suture needle is inserted from the anterior outside of the jejunum about $1 \mathrm{~cm}$ distal to the cut edge and is then withdrawn from the inside of the jejunum lumen. Ligation of the U-suture leads the pancreas stump into the jejunum with no strain on the edge of the pancreas. We experienced no PF in 12 other patients with incomplete invagination of the pancreas stump. And patients who survive for long periods after standard pancreatojejunostomy might be at risk of developing secondary choledochal or pancreatic cancer, as observed in patients with anomalous arrangement of the pancreaticobiliary ductal system [38]. Thus, the SL reconstruction method could potentially prevent both short-term postoperative complications and future secondary carcinogenesis. A greater number of cases must be accumulated to confirm our findings and determine long-term outcomes.

\section{Conclusion}

Certain reports have shown no clear evidence for or against one particular method of pancreaticoenteric anastomosis $[39,40]$. The choice of pancreatic anastomotic method might be based on individual experience and adherence to basic principles such as good exposure and visualization; fine, nonstrangulating suture placement to produce a patent, watertight anastomosis; and preservation of the blood supply [41]. As long as PD is performed, the argument for safety should be continued, and for even non-expert surgeons or in cases of soft pancreas texture, a favorite method that causes the surgeon no anxiety will be chosen. There is still no agreement as to which of the reconstruction methods is best, but early-term

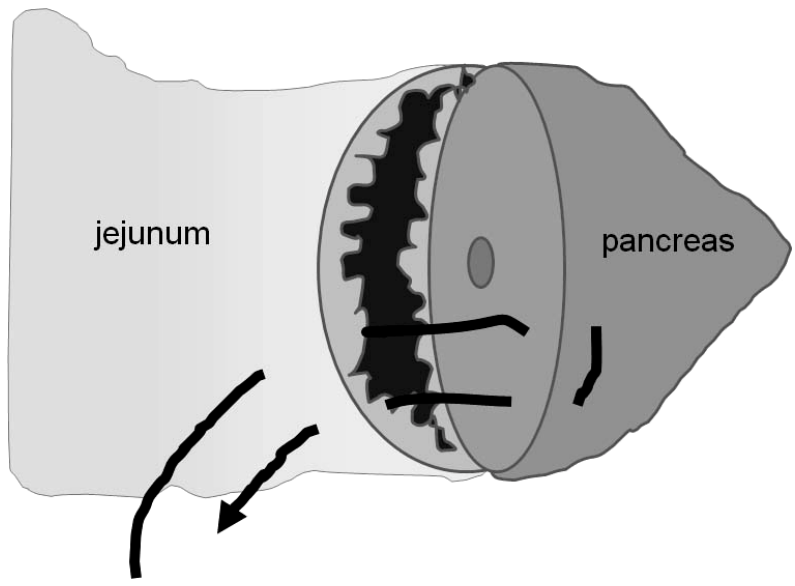

(a)

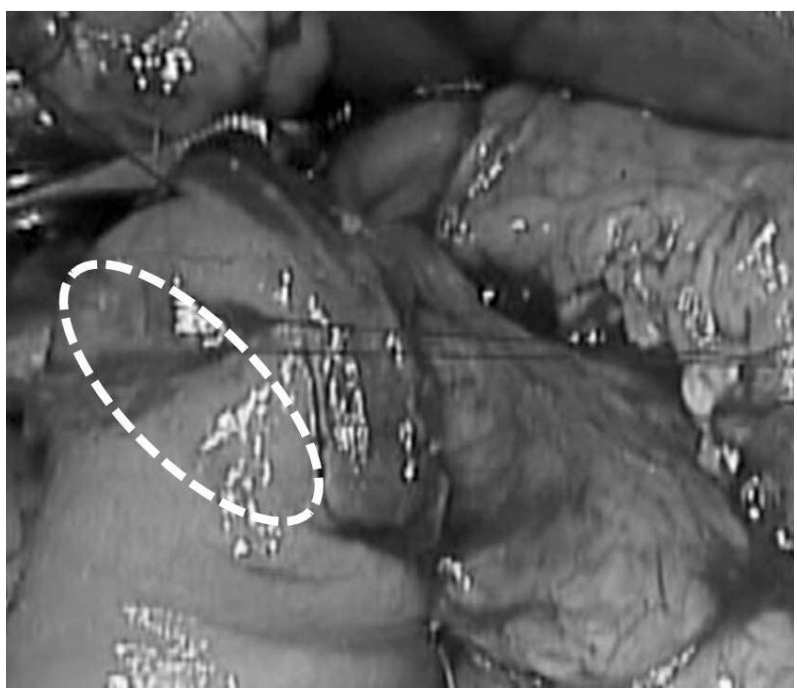

(b)

Figure 2. Recent modified pancreatojejunostomy, incomplete invagination. (Before anterior layer suturing, placed two transpancreatic U-sutures (a). The U-suture was needled from the anterior outside of jejunum about $1 \mathrm{~cm}$ distal to the cut edge, then withdrawing from the inside of the jejunum lumen. Ligation of the $U$-suture leads the pancreas stump (dot area) into the jejunum without any strain for pancreas edge (b)).

observation after PD indicates that the SL method might be superior to the other methods.

\section{References}

[1] J. L. Cameron, T. S. Riall , J. Coleman and K. A. Belcher , "One Thousand Consecutive Pancreaticoduodenectomies,” Annals of Surgery, Vol. 244, No. 1, July 2006, pp. 10-15. doi:10.1097/01.sla.0000217673.04165.ea

[2] M. L. DeOliveira, J. M. Winter, M. Schafer, S. C. Cununingham, J. L. Cameron, C. J. Yeo and P. A. Clavien, "Assessment of Complications after Pancreatic 
Surgery: A Novel Grading System Applied to 633 Patients Undergoing Pancreaticoduodenectomy," Annals of Surgery, Vol. 244, No. 6, December 2006, pp. 931-937. doi:10.1097/01.sla.0000246856.03918.9a

[3] R. T. Poon, S. T. Fan., C. M. Lo, K. K. Ng, W. K. Yuen , C. Yeung, J. Wong, "External Drainage of Pancreatic Duct with a Stent to Reduce Leakage Rate of Panceraticojejunostomy after Pancreaticoduodenectomy," Annals of Surgery, Vol. 246, September 2007, pp. 425-433. doi:10.1097/SLA.0b013e3181492c28

[4] A. Mathur, H. A. Pitt, M. Marine, R. Saxena, C. M. Schmidt, T. J. Howard, A. Nakeeb, N. J. Zyromski and K. D. Lillemoe , "Fatty Pancreas: A Factor in Postoperative Pancreatic Fistula,” Annals of Surgery, Vol. 246, No. 6, December 2007, pp. 1058-1064. doi:10.1097/SLA.0b013e31814a6906

[5] C. Bassi , M. Falconi, E. Molinari, W. Mantovani, G. Butturini, A. A. Gumbs, R. Salvia and P. Pederzoli, "Duct-To-Mucosa Versus End-To-Side Pancreaticojejunostomy Reconstruction after Pancreaticoduodenectomy: Results of a Prospective Randomized Trial,” Surgery, Vol. 134, No. 5, November 2003, pp. 766-771. doi:10.1016/S0039-6060(03)00345-3

[6] S. Hishinuma, Y. Ogata and J. Matsui, "Evaluation of Pylorus-Preserving Pancreatoduodenectomy with the Imanaga Reconstruction by Hepatobiliary and Gastrointestinal Dual Scintigraphy,” The British. Journal of Surgery, Vol. 86, No. 10, October 1999, pp. 1306-1311. doi:10.1046/j.1365-2168.1999.01232.X

[7] S. Hishinuma, Y. Ogata and J. Matsui, "Complications after Pylorus-Preserving Pancreatoduodenectomy with Gastrointestinal Reconstruction by the Imanaga Method,” Journal of the American College Surgeons, Vol. 186, No. 1, January 1998, pp. 10-16. doi:10.1016/S1072-7515(97)00133-6

[8] T. Ohtsuka, K. Yamaguchi, K. Chijiiwa and M. Tanaka, "Effect of Gastrointestinal Reconstruction on Quality of Life and Nutritional Status after Pylorus-Preserving Pancreatoduodenectomy," Digestive Diseases and Sciences, Vol. 47, No. 6, July 2002, pp. 1241-1247. doi:10.1023/A:1015306110913

[9] C. J. Yeo, J. L. Cameron, T. A. Sohn, K. D. Lillemoe, H. A. Pitt, M. A. Talamini, R. H. Hruban, S. E. Ord, P. K. Sauter, J. Coleman, M. L. Zahurak, L. B. Grochow and R. A. Abrams, "Six Hundred Fifty Consecutive Pancreaticoduodenectomies in the 1990s: Pathology, Complications and Outcomes,” Annals of surgery, Vol. 226, No. 3, September 1997, pp. 248-257. doi:10.1097/00000658-199709000-00004

[10] R. Doi, K. Fujimoto, H. Kobayashi and M. Imamura, "Impact of Reconstruction Methods on Outcome of Pancreatoduodenectomy in Pancreatic Cancer Patients," World Journal of Surgery, Vol. 29, No. 4, April 2005, pp. 500-504. doi:10.1007/s00268-004-7723-7

[11] S. Osada, H. Imai, N. Okumura, Y. Tokuyama, Y. Hosono, F. Sakashita and Y. Sugiyama, "A Modified Reconstruction Method to Prevent Critical Complications after Pancreatoduodenectomy,” Hepato-gastroenterology, Vol. 53, No. 8, March/ April 2006, pp. 296-300.
[12] H. Riediger, F. Makowiec, W. D.Schreck , U. T. Hopt and U. Adam "Delayed Gastric Emptying after Pylorus-Pre- Serving Pancreatoduodenectomy Is Strongly Related to the other Postoperative Complications,” Journal of Gastrointestinal Surgery, vol. 7, No. 6, September/October, pp.758-765.

[13] M. Tanakaand M. Sarr, “Total Duodenectomy Effect On Canine Gastrointestinal Motility,” The Journal of Surgical Research, Vol. 42, No. 5, May 1987, pp. 483-493. doi:10.1016/0022-4804(87)90022-9

[14] T. Ohtsuka, K. Kitahara, S. Matsuyama, T. Shimonishi, Y. Nakafusa and K. Miyazaki, "Complication of the Postoperative Outcome after a Pancreatoduodenectomy Using the Billroth I and II Type of Reconstruction," $\mathrm{He}$ pato-gastroenterology, Vol. 54, No. 77, July/August 2007, pp. 1570-1574.

[15] S. Takahata , T. Ohtsuka, T. Nabae, K. Matsunaga, K. Yamaguchi, K. Chijiiwa and M. Tanaka, "Complication of Recovery of Gastric Phase III Motility and Gastric Juice Output after Different Types of Gastrointestinal Reconstruction Following Pylorus-Preserving Pancreatoduodenectomy,” Journal of Gastroenterology Vol. 37, No. 8, August 2002, pp. 596-603. doi:10.1007/s005350200095

[16] S. E. Lee, S. H. Yang, J. Y. Jang and S. W. Kim, "Pancreatic Fistula after Pancreaticoduodenectomy: A Comparison between the Two Pancreaticojejunostomy Methods for Approximating the Pancreatic Parenchyma to the Jejunal Seromuscular Layer: Interrupted Vs. Continuous Stitches," World Journal of Gastroenterology, Vol. 13, No. 4, March 2007, pp. 5351- 5356.

[17] G. P. Fragulidis, N. Arkadopoulos , I. Vassiliou, A. Marinis, T. Thedosopoulos, V. Safyla, M. Kyriazi, K. Karapanos, N. Dafnios, A. Polydorou, D. Voros and V. Smyrniotis, "Pancreatic Leakage after Pancreaticoduodenectomy,” Pancreas, Vol. 38, October 2009, pp. e177-e182. doi:10.1097/MPA.0b013e3181b57705

[18] A. C. Berger, T. J. Howard, E. P. Kennedy, P. K. Sauter, M. Bower-Cherry, S. Dutkevitch, T. Hyslop, C. M. Schmidt, E. L. Rosato, H. Lavu, A. Nakeeb, H. A. Pitt, K. D. Lillemoe and C. J. Yeo, "Does Type of Pancreaticojejunostomy after Pancreaticoduodenectomy Decrease Rate of Pancreatic Fistula? Randomized, Prospective, DualInstitution Trial," Journal of the American College Surgeons, Vol. 208, No. 5, May 2009, pp. 738-749. doi:10.1016/j.jamcollsurg.2008.12.031

[19] J. H. Nguyen, "Distinguishing between Parenchymal and Anastomotic Leakage at Duct-To-Mucosa Pancreatic Reconstruction in Pancreaticoduodenectomy," World Journal of Surgery, Vol. 14, No. 43, November 2008, pp. 6648-6654.

[20] C. Bassi, M. Falconi, E. Molinari, R. Salvia, G. Butturini, N. Sartori, W. Mantovani and P. Pederzoli, "Reconstruction by Pancreaticojejunostomy Versus Pancreaticogastrostomy Following Pancreatectomy: Results of a Comparative Study,” Annals of surgery, Vol. 242, No. 6, December 2005, pp. 767-773.

[21] Y. Murakami, K. Uemura, Y. Hayashidani, T. Sudo, N. Hashimoto, H. Ohge and T. Sueda , "No Mortality after 
150 Consecutive Pancreatoduodenectomies with DuctTo-Mucosa Pancreaticogastrostomy,” Journal of Surgical Oncology, Vol. 97, No. 3, March 2008, pp.205-209. doi:10.1002/jso.20903

[22] J. P. Duffas, B. Suc and S. Msika, "A Controlled Randomized Multicenter Trial of Pancreatogastrostomy or Pancreaticojejunostomy after Pancreatoduodenectomy,” The American Journal of Surgery, Vol. 189, No. 6, July 2005, pp. 720-729. doi:10.1016/j.amjsurg.2005.03.015

[23] M. N. Wente, S. V. Shrikhande, M. W. Muller, M. K. Diener, C. M. Seiler, H. Friess and M. W. Buchler, "Pancreaticojejunostomy Versus Pancreaticogastrostomy: Systemic Review and Meta-Analysis," The American Journal of Surgery, Vol. 193, No. 2, February 2007, pp. 171-183. doi:10.1016/j.amjsurg.2006.10.010

[24] E. Lemaire, D. O’Toole, A. Sauvanet, P. Hammel, J. Belghiti and O. Ruszniewski, "Functional and Morphological Changes in the Pancreatic Remnant Following Pancreaticoduodenectomy with Pancreaticogastric Anastomosis," British Journal of Surgery, Vol. 87, No. 4, April 2000, pp. 434-438. doi:10.1046/j.1365-2168.2000.01388.x

[25] Y. Tomimaru, Y. Takeda, S. Kobayashi, S. Marubashi, C. M. Lee, M. Tanemura, H. Nagao, T. Kitagawa, K. Dono, K. Umeshita, K. Wakasa and M. Monden , "Comparison of Postoperative Morphological Changes in Remnant Pancreas between Pancreaticojejunostomy and Pancreaticogastrostomy after Pancreaticoduodenectomy,” Pancreas, Vol. 38, No. 2, March 2009, pp. 203-207. doi:10.1097/MPA.0b013e31818e1772

[26] U. Adam, F. Makowiec, H. Riedinger, W. D. Schareck, S. Benz and U. T. Hopt, "Risk Factors for Complications after Pancreatic Head Resection," The American Journal of Surgery, Vol. 187, No. 2, February 2004, pp. 201-208. doi:10.1016/j.amjsurg.2003.11.004

[27] N. Akamatsu, Y. Sugawara, M. Komagome, N. Shin, N. Cho, T. Ishida, Ozawa and D. Hashimoto, "Risk Factors for Postoperative Fistula after Pancreaticoduodenectomy: The Significance of the Ratio of the Main Pancreatic Duct to the Pancreas Body as a Predictor of Leakage," Journal of Hepato-Biliary-Pancreatic Sciences, Vol. 17, May 2010, pp. 322-328. doi:10.1007/s00534-009-0248-6

[28] E. Molinari, C. Bassi, R. Salvia, G. Butturini, S. Crippa, G. Talamini, M. Falconi and P. Pederzoli, "Amylase Value in Drain after Pancreatic Resection as Predictive Factor of Postoperative Pancreatic Fistula: Results of a Postoperative Study in 137 Patients," Annals of surgery, vol. 246, No. 2, August 2007, pp. 281-287. doi:10.1097/SLA.0b013e3180caa42f

[29] C. J. Yeo, J. L. Cameron, K. D. Lilemoe, P. K. Sauter, J. Coleman, T. A. Sohn, K. A. Campbell and M. A. Choti , "Does Prophylactic Octreotide Decrease the Rates of Pancreatic Fistula and Other Complications after Pancreaticoduodenectomy? Results of a Prospective Randomized Placebo-Con- Trolled Trial," Annals of surgery, Vol. 232, No. 3, September 2000, pp. 419-429. doi:10.1097/00000658-200009000-00014

[30] A. Ramos-De la Medina and M. G. Starr, "Somatostatin Analogues in the Prevention of Pancreas-Related Com- plications after Pancreatic Resection,” Journal of HepatoBiliary-Pancreatic Surgery, Vol. 13, No. 3, March 2006, pp. 190-193. doi:10.1007/s00534-005-1033-9

[31] S. Connor, N. Alexakis, O. J. Garden, E. Leandros, J. Bramis and S. J. Wigmore, "Meta-Analysis of the Value of Somatostain and Its Analogues in Reducing Complications Associated with Pancreatic Surgery,” British Journal of Surgery, Vol. 92, No. 9, September 2005, pp. 1059-1067. doi:10.1002/bjs.5107

[32] E. Rosso, P. Bachellier, E. Oussoultzoglou, R. Scurtu, N. Meyer, H. Nakano, G. Verasay and D. Jaeck, “Toward Zero Pancreatic Fistula after Pancreaticoduodenectomy with Pancreaticogastrostomy," The American Journal of Surgery, Vol. 191, No. 6, June 2006, pp. 726-732. doi:10.1016/j.amjsurg.2005.09.012

[33] G. V. Aranha , P. Hodul, E. Golts, D. Oh, J. Pickleman and S. Creech, "A Comparison of Pancreaticogastrostomy and Pancreaticojejunostomy Following Pancreaticoduodenectomy," Journal of Gastrointestinal Surgery, Vol. 7, No. 5, August 2003, pp. 672-682. doi:10.1016/S1091-255X(02)00432-8

[34] M. Tani, M. Kawai, S. Hirono, S. Ina, M. Miyazawa, A. Shimizu and H. Yamaue, "A Prospective Randomized Controlled Trial of Internal Versus External Drainage with Pancreaticojejunostomy for Pancreaticoduodenectomy,” The American Journal of Surgery, Vol. 199, No. 6, June 2010, pp. 759-764. doi:10.1016/j.amjsurg.2009.04.017

[35] S. Ohwada, Y. Tanahashi, T. Ogawa, S. Kawate, K. Hamada, K. I. Tago, T. Yamada and Y. Morishita, "In Situ Vs Ex Situ Pancreatic Duct Stents of Duct-To-Mucosa Pancreaticojejunostomy after Pancreaticoduodenectomy with Billroth I-Type Reconstruction," Archives of Surgery, Vol. 137, No. 11, November 2002, pp. 12891293.

[36] M. Kawai, M. Tani, H. Terasawa, S. Ina, S. Hirono, R. Nishioka, M. Miyazawa, K. Uchiyama and H. Yamaue, "Early removal of prophylactic drains reduces the risk of intra-abdominal infections in patients with pancreatic head resection: prospective study for 104 consecutive patients,” Annals of surgery, Vol. 244, No. 1, July 2006, pp. 1-7. doi:10.1097/01.sla.0000218077.14035.a6

[37] S. Osada, Y. Sanada, Y. Tanaka, A. Ikawa, Y. Tokuyama, N. Okumura, Y. Hosono, K. Nonaka, T. Takahashi, K. Yamaguchi and K. Yoshida, "Clinical evaluation of modified reconstruction method after pancreatoduodenectomy," Hepato-gastroenterology, Vol. 56, No. 91-92, May/June 2009, pp. 619-623.

[38] E. Hidaka, A. Yanagisawa, M. Seki, K. Takano, T. Setoguchi and Kato, "High Frequency of K-Ras Mutations in Biliary Duct Carcinoma of Cases with Long Common Channel in the Papilla of Vater," Cancer Research, Vol. 60, No. 522, February 2000, pp. 522-524.

[39] E. C. Lai, S. H. Lau and W. Y. Lau, "Measures to Prevent Pancreatic Fistula after Pancreatoduodenectomy: A Comprehensive Review,” Archives of Surgery, Vol. 144, No. 11, November 2009, pp. 1074-1080.

[40] D. You, K. Jung, H. Lee, J. Heo, S. Choi and D. Choi, 
“Comparison of different pancreatic anastomosis techniques using the definitions of the International Study Group of Pancreatic Surgery: a single surgeon's experience,” Panceas Vol. 38, No. 8, November 2009, pp. 896902. doi:10.1097/MPA.0b013e3181b365f7
[41] D. B. Adams, "The Pancreatic Anastomosis: The Danger of a Leak, Which Anastomotic Technique Is Better?” Journal of Gastrointestinal Surgery, Vol. 13, No. 7, March 2009, pp.1182-1183. doi:10.1007/s11605-009-0865-z 